\title{
Using Difference Scheme Method for the Numerical Solution of Telegraph Partial Differential Equation
}

\author{
Bawar Faraj* and Mahmut Modanli* \\ * Department of Mathematics, Science and Art Faculty, Harran University ,Sanliurfa, Turkey. \\ bawarfaraj@gmail.com and mmodanli@harran.edu.tr
}

\section{Abstract}

In this work, we presented the following hyperbolic telegraph partial differential equation

$$
\left\{\begin{array}{l}
u_{t t}(t, x)+u_{t}(t, x)+u(t, x)=u_{x x}(t, x)+u_{x}(t, x)+f(t, x), 0 \leq t \leq T \\
u(t, 0)=u(t, L)=0, \quad u(0, x)=\varphi(x), u_{t}(0, x)=\Psi(x), \quad 0 \leq x \leq L
\end{array}\right.
$$

Although exact solution of this partial differential equation is known it is important to test reliability of difference scheme method. The Stability estimates for this telegraph partial differential equation are given. The first and second order difference schemes are formed for the abstract form of the above given equation by using initial conditions. Theorem on matrix stability is established for these difference schemes. The first and second order of accuracy difference schemes to approximate solution of this problem are stated. For the approximate solution of this initial-boundary value problem, we consider the set $w_{(\tau, h)}=[0, T]_{\tau} \times[0, L]_{h}$ of a family of grid

points depending on the small parameters $\tau=\frac{T}{N}(N>0)$ and $h=\frac{L}{N}(N>0)$. Gauss elimination method is applied for solving this difference schemes in the case of telegraph partial differential equations. Exact solutions obtained by Laplace transform method is compared with obtained approximation solutions. The theoretical terms for the solution of these difference schemes are supported by the results of numerical experiments. The numerical solutions which found by Matlab program has good results in terms of accuracy. Illustrative examples are included to demonstrate the validity and applicability of the presented technique. As a result, difference scheme method is important for above mentioned equation.

Key Words: Telegraph equation, Numerical Solution, Stability, Finite difference scheme, Error estimate.

\section{Introduction}

The telegraph hyperbolic partial differential equation is important for modeling several relevant problems such as signal analysis, wave propagation, hyperbolic partial differential equations are arise in many branches of mathematics, physics ,other science and engineering, as electrodynamics, thermodynamics, elasticity, wave propagation. in numerical methods for solving these equations, the problem of stability has received a great deal of importance and attention. in recent years, much attention has given in the papers to the solution of a hyperbolic telegraph partial differential equation. Gao and Chi developed a numerical algorithm for the estimate the solution of nonlinear telegraph equations [7]. Dehghan and Shokri present a new numerical scheme (Kansas method) [5] . Koksal showed numerical solutions of the 
telegraph equations with the modified difference scheme [9]. Ashyralev and Modanli present finite difference schemes to find numerical method [1]-[9].The finite difference method is important tool for the solution of telegraph equation.

In the present paper, we consider the following initial-value problem for a telegraph equation

$$
\left\{\begin{array}{l}
u_{t t}(t)+\alpha u_{t}(t)+J u(t)+K u(t)+\beta u(t)=f(t) \\
u(0)=\varphi, u^{\prime}(0)=\psi, \quad 0 \leq t \leq T
\end{array}\right.
$$

in a Hilbert space $H$ with a self-adjoint positive definite operator $J \geq \delta I, K \geq \delta I$ let $A=J+K$, we define an operator $D=J+K+\beta-\frac{\alpha^{2}}{4}$. Here $\delta>0, \alpha>0$ and

$$
\beta+\delta \geq \frac{\alpha^{2}}{4}
$$

A function $u(t)$ is called a solution of problem ( 2 ) if the following conditions are satisfied [3] :

i- The function $u(t)$ is twice continuously differentiable on the segment $[0, T]$..

ii- Element $u(t)$ belongs to $D(A)$ for all $t \in[0, T]$ and the function $D u(t)$ is continuous on the segment $[0, T]$.

iii- $u(t)$ satisfies the equation and initial conditions (2).

Let $\{C(t), t \geq 0\}$ be a strongly continuous cosine operator-function defined in following formula

$$
C(t)=\frac{e^{i \sqrt{D} t}+e^{-i \sqrt{D} t}}{2}
$$

Then, from the definition of the sine operator-function $S(t)$

$$
S(t)=\frac{e^{i \sqrt{D} t}-e^{-i \sqrt{D} t}}{2 i \sqrt{D}}
$$

It is easy to check that under assumption (2) problem (3) has a unique mild solution given by the formula

$$
u(t)=e^{-\frac{\alpha}{2} t}\left[C(t)+\frac{\alpha}{2} S(t)\right] u_{0}+e^{-\frac{\alpha}{2} t}[S(t)] u_{0}^{\prime}+\int_{0}^{t} e^{-\frac{\alpha}{2}(t-s)} S(t-s) f(s)
$$

following theorem shows the stability of problem (2).

Theorem 1. [1] Suppose that $\varphi \in D(A), \psi \in D\left(A^{\frac{1}{2}}\right), A=J+K$ and $f(t)$ is a continuously differentiable function on $[0, T]$ and the assumption (3) holds. Then, there is a unique solution of problem (2) and the stability inequalities.

$$
\max _{0 \leq t \leq T}\|u(t)\|_{H}
$$




$$
\begin{gathered}
\leq M(\alpha, \beta, \delta)\left[\|\varphi\|_{H}+\left\|A^{-\frac{1}{2}} \psi\right\|_{H}+\max _{0 \leq t \leq T}\left\|A^{-\frac{1}{2}} f(t)\right\|_{H}\right], \\
\max _{0 \leq t \leq T}\left\|\frac{d u(t)}{d t}\right\|_{H}+\max _{0 \leq t \leq T}\left\|A^{\frac{1}{2}} u(t)\right\|_{H} \\
\leq M(\alpha, \beta, \delta)\left[\left\|A^{\frac{1}{2}} \varphi\right\|_{H}+\|\psi\|_{H}+\max _{0 \leq t \leq T}\|f(t)\|_{H}\right], \\
\max _{0 \leq t \leq T}\left\|\frac{d^{2} u(t)}{d t^{2}}\right\|_{H}+\max _{0 \leq t \leq T}\|A u(t)\|_{H} \\
\leq M(\alpha, \beta, \delta)\left[\|A \varphi\|_{H}+\left\|A^{\frac{1}{2}} \psi\right\|_{H}+\|f(0)\|_{H}+\max _{0 \leq t \leq T}\left\|f^{\prime}(t)\right\|_{H} d t\right]
\end{gathered}
$$

where $M(\alpha, \beta, \delta)$ does not depend on $\varphi, \psi$ and $f(t)$.

The aim of this study is to construct and investigate the difference schemes for the telegraph equation.

\section{Stability of Difference Scheme}

For the approximate solution of initial value problem (2), we consider the grid space $[0, T]_{\tau}=\left\{t_{k}=k \tau, 0 \leq k \leq N, \tau N=T\right\}$ By using the method in [3] we get the first order difference scheme

$$
\left\{\begin{array}{l}
\frac{u_{k+1}-2 u_{k}+u_{k-1}}{\tau^{2}}+\alpha \frac{u_{k+1}-u_{k-1}}{2 \tau}+(J+K+\beta) u_{k}=f_{k} \\
u(0)=\varphi, \quad \frac{u_{1}-u_{0}}{\tau}+\left(J+K+\left(\beta-\frac{\alpha^{2}}{4}\right) I\right) \tau u_{1}=\frac{1}{1+\frac{\alpha}{2} \tau} \psi
\end{array}\right.
$$

and second order difference scheme

$$
\left\{\begin{array}{l}
\frac{u_{k+1}-2 u_{k}+u_{k-1}}{\tau^{2}}+\alpha \frac{u_{k+1}-u_{k-1}}{2 \tau}+(\beta+J+K) \frac{u_{k+1}+u_{k-1}}{2}=f_{k} \\
u(0)=\varphi \\
\frac{u_{\tau}-u_{0}}{\tau}+\frac{\tau}{4} D u_{1}+\frac{1}{1+\frac{\alpha}{4} \tau}\left(\frac{1}{4} D-\frac{\alpha \tau D}{16}+\frac{\alpha^{2}}{8} I\right)+\tau u_{1}=\frac{1-\frac{\alpha}{4} \tau}{1+\frac{\alpha}{4} \tau}\left(\psi+\frac{\tau}{2} f_{0}\right)
\end{array}\right.
$$

Similarity to [11], the stability of first order difference scheme in ( 7$)$ we define $S\left(t_{k}\right)=J+K+\beta, P\left(t_{k}\right)=\alpha\left(t_{k}\right)$ and $Q\left(t_{k}\right)=\frac{1}{\tau^{2}}$ we get 


$$
Q\left(t_{k}\right)\left(u_{k+1}-2 u_{k}+u_{k-1}\right)+P\left(t_{k}\right) \frac{u_{k+1}-u_{k-1}}{2 \tau}+S\left(t_{k}\right) u_{k}=f\left(t_{k}\right)
$$

From here we instead of the difference scheme in (8) for homogeneous part of the following difference scheme

$$
Q\left(u_{k+1}-2 u_{k}+u_{k-1}\right)+P \frac{u_{k+1}-u_{k-1}}{2 \tau}+S u_{k}=0
$$

The following theorem guaranty the stability of (7).

Theorem 2. [11] Let the operators $Q$ and $S$ in the operator difference scheme (10) be self adjoint operators, then with the conditions

$$
P \geq 0 \quad S>0 \quad Q>\frac{1}{4} S
$$

fulfilled, there holds the a priori estimate

$$
\begin{aligned}
\frac{1}{4}\left\|u_{k+1}+u_{k}\right\|_{S}^{2} & +\left\|u_{k+1}-u_{k}\right\|_{Q}^{2}-\frac{1}{4}\left\|u_{k+1}-u_{k}\right\|_{S}^{2} \\
& \leq \frac{1}{4}\left\|u_{k}+u_{k-1}\right\|_{S}^{2}+\left\|u_{k}-u_{k-1}\right\|_{Q}^{2}-\frac{1}{4}\left\|u_{k}-u_{k-1}\right\|_{S}^{2}
\end{aligned}
$$

Where $S, Q$ are self adjoint difference operators, $H$ denoted a Hilbert space $\|.\|_{H}$ is norm in Hilbert space, $\|.\|_{S}$ norm in $H_{S}\|.\|_{Q}$ norm in $H_{Q}$.

\section{Numerical Computation}

In applications, let us consider the initial-boundary value problem hyperbolic telegraph partial differential equation

$\left\{\begin{array}{l}u_{t t}(t, x)+u_{t}(t, x)+u(t, x)=u_{x x}(t, x)+u_{x}(t, x)+f(t, x), 0 \leq t \leq T \\ u(t, 0)=u(t, L)=0, \quad u(0, x)=\varphi(x), u_{t}(0, x)=\Psi(x), 0 \leq x \leq L\end{array}\right.$

Where $\Psi(x), \varphi(x)(x \in[0, \pi])$ and $f(t, x),((t, x) \in[0,1] \times[0, \pi])$ are smooth functions, problem (12) presents a damped wave equation and a telegraph equation [3].To find difference scheme for (12) we use Taylor expansion and we have $\left(t_{k-1}, t_{k}, t_{k+1}\right)$ and $\left(x_{n-1}, x_{n}, x_{n+1}\right)$ gird points to find difference scheme, here we have the following examples

$$
\left\{\begin{array}{l}
u_{t t}(t, x)+u_{t}(t, x)-u_{x x}(t, x)-u_{x}(t, x)+u(t, x)=(2 \sin (x)-\cos (x)) e^{-t} \\
u(0, x)=\sin x, u_{t}(0, x)=-\sin x, \quad u(t, 0)=u(t, \pi)=0 \\
\text { where } 0<t<1,0<x<\pi
\end{array}\right.
$$

it has an exact solution $u(x, t)=\sin (x) e^{-t}$ established by Laplace transform, for above example we have the first order of difference scheme 


$$
\left\{\begin{array}{l}
\frac{u_{n}^{k+1}-2 u_{n}^{k}+u_{n}^{k-1}}{\tau^{2}}+\frac{u_{n}^{k+1}-u_{n}^{k}}{\tau}-\frac{u_{n+1}^{k+1}-2 u_{n}^{k+1}+u_{n-1}^{k+1}}{h^{2}}-\frac{u_{n+1}^{k+1}-u_{n}^{k+1}}{h} \\
+u_{n}^{k+1}=\left(2 \sin \left(x_{n}\right)-\cos \left(x_{n}\right)\right) e^{-t_{k+1}} \\
u_{n}^{0}=\sin \left(x_{n}\right), \quad \frac{u_{n}^{1}-u_{n}^{0}}{\tau}=-\sin \left(x_{n}\right), u_{0}^{k}=u_{\pi}^{k}=0
\end{array}\right.
$$

and the second order of difference scheme is

$$
\left\{\begin{array}{l}
\frac{u_{n}^{k+1}-2 u_{n}^{k}+u_{n}^{k-1}}{\tau^{2}}+\frac{u_{n}^{k+1}-u_{n}^{k-1}}{2 \tau}-\frac{1}{2} \frac{u_{n+1}^{k+1}-2 u_{n}^{k+1}+u_{n-1}^{k+1}}{h^{2}}-\frac{1}{2} \frac{u_{n+1}^{k-1}-2 u_{n}^{k-1}+u_{n-1}^{k-1}}{h^{2}} \\
-\frac{1}{4} \frac{u_{n+1}^{k+1}-u_{n-1}^{k+1}}{h}-\frac{1}{4} \frac{u_{n+1}^{k-1}-u_{n-1}^{k-1}}{h}+\frac{1}{2} u_{n}^{k+1}+\frac{1}{2} u_{n}^{k-1}=\left(2 \sin \left(x_{n}\right)-\cos \left(x_{n}\right)\right) e^{-t_{k}} \\
u_{n}^{0}=\sin \left(x_{n}\right), \quad \frac{u_{n}^{1}-u_{n}^{0}}{\tau}=-\sin \left(x_{n}\right)+\frac{\tau}{2} \frac{u_{n}^{2}-2 u_{n}^{1}+u_{n}^{0}}{\tau^{2}}, u_{0}^{k}=u_{\pi}^{k}=0
\end{array}\right.
$$

Second example is

$$
\left\{\begin{array}{l}
u_{t t}(t, x)+u_{t}(t, x)-u_{x x}(t, x)-u_{x}(t, x)+u(t, x)=(4 \sin (x)-\cos (x)) e^{t} \\
u(0, x)=\sin x, u_{t}(0, x)=\sin x, \quad u(t, 0)=u(t, \pi)=0 \\
\text { where } 0<t<1,0<x<\pi
\end{array}\right.
$$

with exact solution $u(x, t)=\sin (x) e^{t}$

for above example we have the first order of difference scheme as

$$
\left\{\begin{array}{l}
\frac{u_{n}^{k+1}-2 u_{n}^{k}+u_{n}^{k-1}}{\tau^{2}}+\frac{u_{n}^{k+1}-u_{n}^{k}}{\tau}-\frac{u_{n+1}^{k+1}-2 u_{n}^{k+1}+u_{n-1}^{k+1}}{h^{2}}-\frac{u_{n+1}^{k+1}-u_{n}^{k+1}}{h} \\
+u_{n}^{k+1}=\left(4 \sin \left(x_{n}\right)-\cos \left(x_{n}\right)\right) e^{t_{k+1}} \\
u_{n}^{0}=\sin \left(x_{n}\right), \quad \frac{u_{n}^{1}-u_{n}^{0}}{\tau}=\sin \left(x_{n}\right), u_{0}^{k}=u_{\pi}^{k}=0
\end{array}\right.
$$

and second order of difference scheme is

$$
\left\{\begin{array}{l}
\frac{u_{n}^{k+1}-2 u_{n}^{k}+u_{n}^{k-1}}{\tau^{2}}+\frac{u_{n}^{k+1}-u_{n}^{k-1}}{2 \tau}-\frac{1}{2} \frac{u_{n+1}^{k+1}-2 u_{n}^{k+1}+u_{n-1}^{k+1}}{h^{2}}-\frac{1}{2} \frac{u_{n+1}^{k-1}-2 u_{n}^{k-1}+u_{n-1}^{k-1}}{h^{2}} \\
-\frac{1}{4} \frac{u_{n+1}^{k+1}-u_{n-1}^{k+1}}{h}-\frac{1}{4} \frac{u_{n+1}^{k-1}-u_{n-1}^{k-1}}{h}+\frac{1}{2} u_{n}^{k+1}+\frac{1}{2} u_{n}^{k-1}=\left(4 \sin \left(x_{n}\right)-\cos \left(x_{n}\right)\right) e^{t_{k}} \\
u_{n}^{0}=\sin \left(x_{n}\right), \quad \frac{u_{n}^{1}-u_{n}^{0}}{\tau}=\sin \left(x_{n}\right)+\frac{\tau}{2} \frac{u_{n}^{2}-2 u_{n}^{1}+u_{n}^{0}}{\tau^{2}}, u_{0}^{k}=u_{\pi}^{k}=0
\end{array}\right.
$$

We have $(N+1) \times(M+1)$ system of linear equations and we will write it in the matrix form

$$
A U_{n+1}+B U_{n}+C U_{n-1}=D \varphi(n) \quad 1 \leq n \leq M, u_{0}=u_{M}=0 .
$$


To solve difference scheme in (19) modified Gauss elimination method was used. Hence, we look for a solution of the matrix equation in the following form:

$$
u_{j}=\alpha_{j+1} u_{j+1}+B_{j+1}, u_{M}=0, j=M-1, \ldots, 2,1 .
$$

Where $\alpha_{j}(j=1,2, \ldots, M)$ are $(N+1) \times(N+1)$ is a square matrix, and $\beta_{j}(j=$ $1,2, \ldots, M)$ are $(N+1) \times 1$ column matrices defined by

$$
\begin{gathered}
\alpha_{j+1}=-\left(B+C \alpha_{j}\right)^{-1} A, \\
\beta_{j+1}=\left(B+C \alpha_{j}\right)^{-1}\left(D \phi-C \beta_{j}\right), j=1,2, \ldots, M-1
\end{gathered}
$$

where $j=1,2, \ldots, M-1, \alpha_{1}$ is the $(N+1) \times(N+1)$ zero matrix, and $\beta_{1}$ is the $(N+1) \times 1$ zero matrix. The results of calculations tell us the second order difference schemes has a more accuracy than first order of difference scheme., the following table shows the maximum error accuracy of finite difference scheme by comparison between the numerical solution and exact solution in different values of $N$ and $M$ the maximum error was showed where $N=1 / \tau$ and $M=1 / h$. The errors are computed by

$$
E_{M}^{N}=\max _{1 \leq k \leq N .1 \leq n \leq M-1}\left|u\left(t_{k}, x_{n}\right)-u_{n}^{k}\right|,
$$

Where $u\left(t_{k}, x_{n}\right)$ symbolizes the exact solution and $u_{n}^{k}$ symbolizes the numerical solution at $\left(t_{k}, x_{n}\right)$ and the result are given in the following tables.

For the first example maximum error analysis are

\begin{tabular}{|c|c|c|c|c|}
\hline Method & $\mathrm{N}=\mathrm{M}=25$ & $\mathrm{~N}=\mathrm{M}=50$ & $\mathrm{~N}=\mathrm{M}=100$ & $\mathrm{~N}=\mathrm{M}=150$ \\
\hline $\begin{array}{c}\text { First Order } \\
\text { Difference Scheme }\end{array}$ & $1.2258 \times 10^{-2}$ & $6.3671 \times 10^{-3}$ & $3.2491 \times 10^{-3}$ & $2.1801 \times 10^{-3}$ \\
\hline $\begin{array}{c}\text { Second Order } \\
\text { Difference Scheme }\end{array}$ & $4.5920 \times 10^{-4}$ & $1.1560 \times 10^{-4}$ & $2.8997 \times 10^{-5}$ & $1.2901 \times 10^{-5}$ \\
\hline
\end{tabular}

For second example maximum errors are

\begin{tabular}{|c|c|c|c|c|}
\hline Method & $\mathrm{N}=\mathrm{M}=25$ & $\mathrm{~N}=\mathrm{M}=50$ & $\mathrm{~N}=\mathrm{M}=100$ & $\mathrm{~N}=\mathrm{M}=150$ \\
\hline $\begin{array}{c}\text { First Order } \\
\text { Difference Scheme }\end{array}$ & $8.4964 \times 10^{-2}$ & $4.3329 \times 10^{-2}$ & $2.1858 \times 10^{-2}$ & $1.4615 \times 10^{-2}$ \\
\hline $\begin{array}{c}\text { Second Order } \\
\text { Difference Scheme }\end{array}$ & $6.3553 \times 10^{-4}$ & $1.5960 \times 10^{-4}$ & $4.0048 \times 10^{-5}$ & $1.7811 \times 10^{-5}$ \\
\hline
\end{tabular}

\section{Conclusion}

In the presented paper, we discussed the Cauchy problem for the telegraph equations in (1) . first order and second order of difference scheme for the Cauchy problem are showed, stability for cauchy problem and difference scheme are 
established. The difference schemes of the first order and second order of accuracy for telegraph partial differential equations are showed. Two test example is given, the numerical solution was established, numerical and exact solutions are compared. The comparison tell us the difference scheme method of the second order of approximation gives better result than the first order. Numerical results are obtained using Matlab. The theoretical statements for the solution of these difference schemes are supported by the numerical results.

\section{References}

[1] A.Ashyralyev, and M.Akat, 'AIP Conference Proceedings' 1479,598-601 (2012).

[2] A.Ashyralyev and M.Modanli, 'An operator method for telegraph partial differential and difference equations', Boundary Value Problems, 2015(1), $1-17$.

[3] A.Ashyralyev, M.Modanli, 'A numerical solution for a telegraph equation', AIP Conference Proceedings, 300-304, Doi: 10.1063/1.4893851.

[4] A.Ashyralyev and P.E.Sobolevskii, 'New Difference Schemes for Partial Differential Equations', Birkhauser Verlag, Basel, Boston, Berlin, 2004.

[5] M.Dehghan, and A.Shokri, ' Numerical Methods for Partial Differential Equations' 24, 1080-1093 (2008).

[6] F.Gao and C.Chi, 'Unconditionally stable Difference Scheme for a one- space dimensional linear hyperbolic equation', Appl. Math. Comput., Vol. 187, pp.1272 1276, 2007.

[7] F.Gao, and C.Chi, 'Applied Mathematics and Computation' 187, 1272-1276 (2007).

[8] Grcar, F.Joseph, 'Mathematicians of Gaussian elimination', Notices of the American Mathematical Society, (2011b) 58 (6): 782-792.

[9] M.Koksal, 'Discrete Dynamics in Nature and Society' 2011, 1-17 (2011), Article ID561015.

[10] A.Samaraski, 'The Theory of Difference Schemes, Advances and Applications'. Birkhauser, Basel (2004) ISBN: 0-8247-0468-1.

[11] A.Samaraski, P.N.Vabishechevich,'Numerical Methods for solving inverse problems of mathematical physics', walter de Gruyter,New york(2007). 